\title{
Efecto de Dos Probióticos que Contienen Cepas de Lactobacillus casei variedad rhamnosus y Lactobacillus johnsonii sobre el Crecimiento in Vitro de Streptococcus mutans
}

\author{
Effect of Two Probiotics Containing Lactobacillus casei rhamnosus and \\ Lactobacillus johnsonii variety on the in vitro Growth of Streptococcus mutans
}

\author{
M. Rebolledo*; E. Rojas* \& F. Salgado*
}

REBOLLEDO, M.; ROJAS, E. \& SALGADO, F. Efecto de dos probioticos que contienen cepas de Lactobacillus casei variedad rhamnosus y Lactobacillus johnsonii sobre el crecimiento in vitro de Streptococcus mutans. Int. J. Odontostomat., 7(3):415-419, 2013.

RESUMEN: La caries dental es una de las enfermedades mas prevalentes en la población mundial y más aún en los países en desarrollo, por lo que se necesitan nuevas medidas para su prevención. Estudios previos señalan que el efecto de cepas probióticas inhibe la colonización de los patógenos responsables de la caries dental como el Streptococcus mutans, previniendo la aparición de la caries dental. El objetivo fue medir el efecto de las cepas probióticas Lactobacillus casei variedad rhamnosus (LCR32) y Lactobacillus johnsonii (LA1) sobre le crecimiento in vitro de Streptococcus mutans. Se midió el efecto in vitro de las cepas de dos probióticos comercializados en Chile; Lactobacillus casei variedad rhamnosus (LCR32) contenidas en Lactil 8 y Lactobacillus johnsonii (LA1) contenidas en Chamyto, sobre el crecimiento in vitro de Streptococcus mutans. Se realizaron medios de cultivo selectivos para Streptococcus mutans a los cuales se les adicionaron cuatro diluciones diferentes de cada probiótico y se midió el halo de inhibición de los Streptococcus mutans con un pie de metro. Los probióticos con las cepas Lactobacillus casei variedad rhamnosus (LCR32) y Lactobacillus johnsonii (LA1) inhiben el crecimiento sobre Streptococcus mutans. Ambos probióticos en las dos concentraciones más altas no obtuvieron diferencias significativas en relación a los halos de inhibición. Sin embargo, el probiótico con la cepa Lactobacillus casei variedad rhamnosus (LCR35), mostró halos de inhibición más significativos en comparación a la cepa Lactobacillus johnsonii (LA1). Las cepas probióticas Lactobacillus casei variedad rhamnosus (LCR35) y Lactobacillus Johnosonii (LA1) disminuyen la colonización de las principales bacterias productoras de caries dental, de tal forma estos probióticos podrían ser utilizados como apoyo en la prevención y profilaxis de la enfermedad en pacientes de alto riesgo cariogénico, en forma adicional a otros medios de prevención.

PALABRAS CLAVE: caries, probióticos, cepas bacterianas, Lactobacillus, Streptococcus mutans.

\section{INTRODUCTION}

La caries dental es un proceso multifactorial que provoca la desmineralización y subsecuente cavitación de los dientes, producto de la presencia de un alto número de bacterias cariogénicas, especialmente el Streptococcus mutans. Otros factores asociados a este proceso como la dieta, el huésped y el tiempo, son responsables de su aparición; si son interceptados, pueden detener la progresión de la enfermedad (Teughels et al., 2008). Esta enfermedad es frecuente en países en desarrollo como Chile, en donde el acceso a atención dental es limitada y se necesita desarrollar de nuevas medidas preventivas de bajo costobeneficio como el uso de probióticos.

El término probiótico fue definido en 2002 por la Organización mundial de la Salud (FAO/WHO) como: "microorganismos vivos que al ser administrados en cantidades suficientes promueven efectos fisiológicos

\footnotetext{
* Facultad de Odontología, Universidad del Desarrollo, Concepción, Chile.

* Biólogo, Magister en microbiología. Facultad de Odontología, Universidad del Desarrollo, Concepción, Chile.
} 
beneficiosos sobre el huésped" (Araya et al., 2002). Se cree que los probióticos actúan a través de una variedad de mecanismos, como la competencia con los posibles agentes patógenos, degradación de toxinas,producción de sustancias antimicrobianas e inmunomoduladores locales y sistémicos (Meurman \& Stamatova, 2007). Estos mecanismos de acción se han obtenido exclusivamente a partir de estudios gastrointestinales, creando la necesidad de su aplicación en estudios relacionados con la salud oral. La boca representa la primera parte del tracto gastrointestinal, por lo tanto, hay razones para creer que al menos algunos mecanismos de los probióticos pueden desempeñar un papel importante a nivel oral (Meurman, 2005). El número de productos que contienen probióticos ha aumentado, aunque algunas especies contenidas en estos se asocian a la progresión de caries dental como los Lactobacillus $s p$., que a pesar de ser productoras de acido láctico la capacidad amortiguadora de los productos lácteos que las contienen contrarresta la acidez protegiendo la superficie dental e inhibiendo la adhesión del Streptococcus mutans (Pérez-Luyo, 2008). Al mismo tiempo los Lactobacillus han sido los de mayor interés en la investigación dental por varias décadas, desarrollándose modernas técnicas moleculares en las que estas bacterias se asocian más en las etapas tardías que al inicio del proceso de las lesiones de caries dental (Twetman \& Stecksén-Blicks, 2008).

Actualmente, los probióticos son utilizados como agentes bioterapéuticos por los potenciales beneficios que otorgan al organismo, principalmente a nivel digestivo y del sistema inmune. Al poseer características que los hacen ideales para su consumo, ya que son inocuos, no colonizan en forma permanente el tracto digestivo, actúan como inmunomoduladores y se mantienen vivos por largos periodos de tiempo.

En esta investigación, se evaluó el efecto de dos probióticos Chamyto y Lacti comercializados en Chile, sobre el crecimiento in vitro de los Streptococcus mutans, cuyos resultados aportarían al desarrollo en la prevención de caries dental en pacientes con alto riesgo cariogénico.

\section{MATERIAL Y MÉTODO}

Se realizó un diseño de tipo experimental, utilizando el método de difusión de agar estandarizado, de uso común en el laboratorio de microbiología. Este método se basa en la obtención de resultados cuantitativos obtenidos mediante la determinación de la concentración mínima inhibitoria (Cona, 2010).

Se utilizaron dos cepas probióticas que se obtuvieron de los productos "Lacti|ß" (Laboratorio Chile) que contiene la cepa Lactobacillus casei variedad rhamnosus (LCR35) liofilizada, en una proporción de 108 UFC en $1 \mathrm{~g}$, probiótico administrado vía oral disuelto en agua y "Chamyto" (Nestlé), que contiene Lactobacillus johnsonii (La1), en una proporción de 107 UFC por ml, probiótico administrado por vía oral.

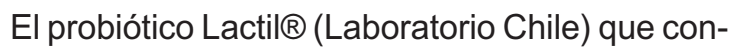
tiene la cepa Lactobacillus casei variedad rhamnosus (LCR32) liofilizada, fue rehidratada en un tubo de ensayo con $5 \mathrm{ml}$ de agua bi destilada, durante $24 \mathrm{~h}$.

Se cultivó la cepa Lactobacillus casei variedad rhamnosus (LCR32) hidratada en medio de cultivo BHI, en condiciones de microaerofilia durante $24 \mathrm{~h}$ para comprobar su viabilidad.

Se prepararon 15 placas Agar Mitis Salivarius (BIOMARK). Se cultivaron los Streptococcus mutans 3sp 5209 del cepario en 5 placas de Agar Mitis Salivarius con $20 \%$ de sacarosa en condiciones de microaerofilia. Después de $24 \mathrm{~h}$ se ajustaron al patrón 0,5 McFarland; se preparo con el siguiente protocolo: Una alícuota de $0,5 \mathrm{ml}(0,048 \mathrm{~m} / \mathrm{l})$ de $\mathrm{BaCL}_{2}(1,175 \%$ $\left.\mathrm{p} / \mathrm{v} \mathrm{BaCL} \mathrm{B}_{2} \times 2 \mathrm{H}_{2} \mathrm{O}\right)$ se agrego 99,5 $\mathrm{ml} \mathrm{de}_{2} \mathrm{SO}_{4}(0,12$ $\mathrm{mol} / \mathrm{L})(1 \% \mathrm{v} / \mathrm{v})$ manteniéndolo en el agitador Vortex por $10 \mathrm{~s}$ para mantener la turbidez correcta).

A partir de $5 \mathrm{ml}$ de Lactil $囚$ y Chamyto contenidos en un tubo de ensayo (dilución directa) se realizaron diluciones seriadas $1 / 10,1 / 100$ y $1 / 1000$, obteniéndose cuatro diluciones distintas para cada probiótico a partir de la directa (1). Estas se realizaron a partir de $1 \mathrm{ml}$ de probiótico y $9 \mathrm{ml}$ de suero fisiológico, cada una agitada previamente en agitador Vortex V1 (Plus msh300).

Cada una de las 8 placas de Agar cultivadas se perforaron en 3 puntos con sacabocado estéril, de tal manera de obtener pocillos de siembra y 2 placas se perforaron en 2 puntos, en un pocillo se aplicó un control positivo y uno negativo, clorhexidina $0,12 \%$ y suero fisiológico respectivamente.

Los Streptococcus mutans que se ajustaron al patrón 0,5 McFarland se tomaron $100 \mathrm{ml}$ con micropipetas y puntas estériles, para ser llevadas a 
las 10 placas de Agar Mitis salivarius perforadas, se sembraron con cotones estériles cada una de las placas y al mismo tiempo en cada placa de agar perforada con un pocillo, se adicionó una alícuota de $80 \mathrm{ml}$ de cada una de tres diluciones distintas (directa, 1/10, $1 / 100$ y 1/1000) de cada probiótico previamente pasadas por agitador Vortex.

Las 10 placas fueron cultivadas por un periodo de $48 \mathrm{~h}$ en condiciones de microaerofilia en jarras de Gaspack y se llevaron a la estufa a $37,8^{\circ} \mathrm{C}$. Entre las 24 y 48 h se midió con pie de metro el halo de inhibición para las cepas de Lactobacillus casei variedad rhamnosus (LCR35) y Lactobacillus johnsonii (LA1). El experimento se realizó en triplicado.
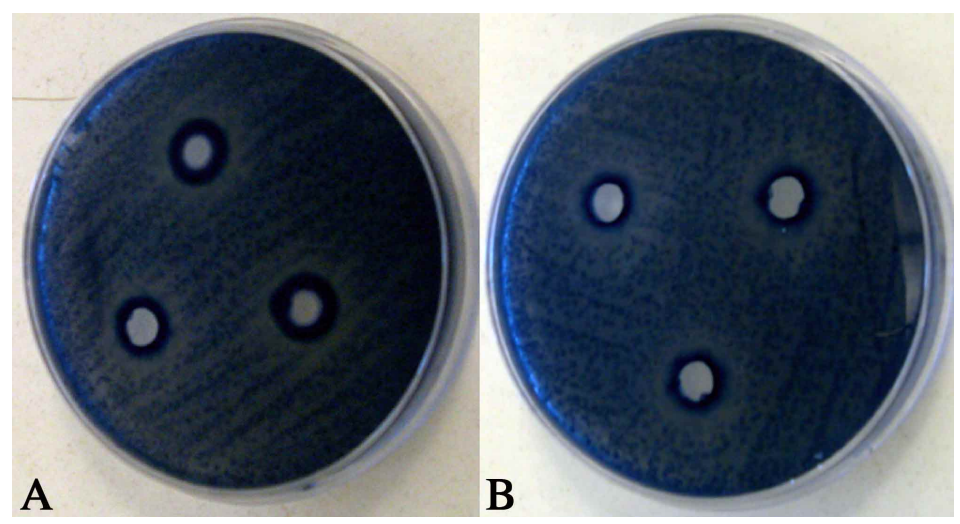

Fig. 1. Placas con Chamyto (A) y Lactil (B) en dilución directa.

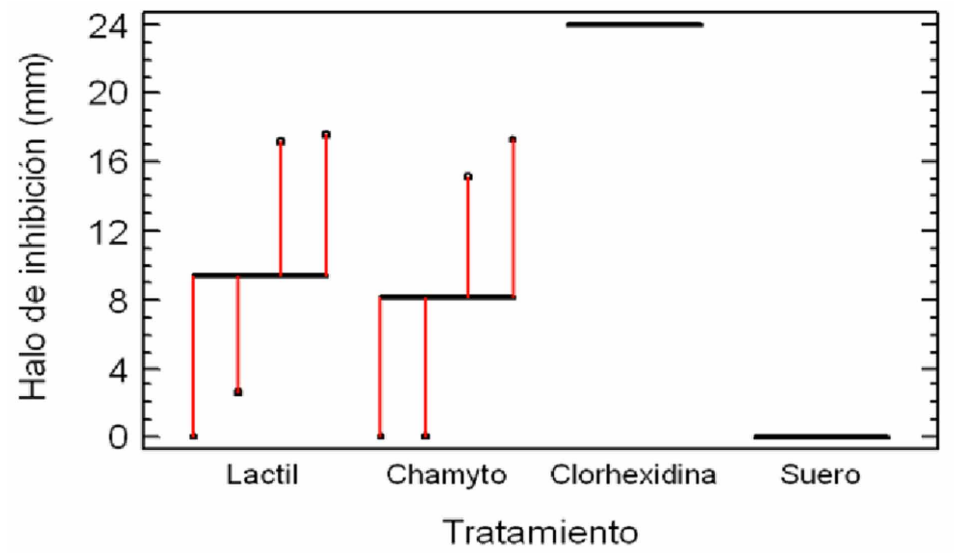

Fig. 2. Halos de inhibición para los cuatro tratamientos.

Tabla I. Valores promedio de concentración para cada probiótico (Media $\pm \mathrm{DE})$.

\begin{tabular}{lcccc}
\hline & 1 & $1 / 10$ & $1 / 100$ & $1 / 1000$ \\
\hline Lactil & $17,5 \pm 3,0$ & $17,2 \pm 2,1$ & $2,6 \pm 0,0$ & $0,0 \pm 0,0$ \\
\hline Chamyto & $17,2 \pm 2,7$ & $15,2 \pm 3,8$ & $0,0 \pm 0,0$ & $0,0 \pm 0,0$ \\
\hline
\end{tabular}

\section{RESULTADOS}

En este estudio dos cepas de bacterias contenidas en dos probióticos fueron analizadas en cuatro diluciones distintas. Los Streptococcus mutans que se ajustaron a patrón 0,5 McFarland, se inocularon en medio selectivo Agar mitis salivarius que contenían los pocillos de siembra con las cepas probióticas Lactobacillus casei variedad rhamnosus (LCR35) y Lactobacillus johnsonii (LA1) en; dilución 1 (directa), $1 / 10,1 / 100$ y $1 / 1000$, resultando diferentes halos de inhibición en $\mathrm{mm}$ después de $24 \mathrm{~h}$ (Fig. 1). Se obtuvieron los resultados mostrados en la Tabla I y expresados en el gráfico (Fig. 2).

El valor de significancia entre ambos probióticos Lactil y Chamyto fue de un $p=0,82$ y entre concentraciones para Lactil y Chamyto un $p=0,00$.

\section{DISCUSIÓN}

En el presente estudio se mostraron los halos de inhibición sobre los Streptococcus mutans de dos probióticos que contenían las cepas Lactobacillus casei variedad rhamnosu (LCR35) y Lactobacillus johnsonii (LA1), en distintas concentraciones. De acuerdo al análisis no existen diferencias significativas entre los valores medios de ambos probióticos ya que se obtuvo un resultado de $p=0,082(p>0,05)$. Sin embargo si existen diferencias significativas entre las concentraciones dentro de cada probiótico ya que se obtuvo un $p=0.00$ $(p<0.05)$.

Esto quiere decir que para ambos probióticos en concentraciones 1 y $1 / 10$ no obtuvieron diferencia significativas entre si, al igual que las concentraciones $1 / 100$ y $1 /$ 1000. Sin embargo si se obtuvieron diferencias significativas entre las dos primeras y las dos segundas, siendo el probióticos con la cepa Lactobacillus casei variedad rhamnosus (LCR35), la que mostró halos de inhibición más grandes en comparación a la cepa Lactobacillus johnsonii (LA1).

Todas las concentraciones para am- 
bos probióticos se realizaron a partir de dosis terapéuticas por lo tanto su uso nos haría pensar que efectivamente podría causar algún cambio sobre microflora oral al momento de consumirlos.

Los resultados obtenidos en este estudio se relacionan con los obtenidos por Ahola et al. (2002), Nikawa et al. (2004) y Caglar et al. (2006), quienes usaron probióticos con cepas de Lactobacillus que disminuyeron la cuenta de Streptococcus mutans después de haber usado distintos medios de administración, durante un tiempo determinado y en grupos etáreos diferentes. En un estudio hecho por Caglar et al. (2005) se concluyó que los sujetos que consumen diariamente bioyogurt con $L$. rhamnosus GG, albergaron a este microorganismo en su saliva hasta dos semanas después de suspender el consumo de los probióticos. Meurman demostró que el consumo a largo plazo de algunos probióticos reduce significativamente el riesgo de caries en niños, ya que estos podrían colonizar temporalmente la cavidad oral.

Sería de gran utilidad estudiar los mecanismos por los cuales actúan estas cepas sobre los Streptococcus mutans y sus posibles efectos in vitro, realizar estudios in vivo para determinar el tiempo que estas bacterias contenidas en probióticos son capaces de colonizar la cavidad oral, al mismo tiempo realizar estudios sobre las cepas probióticas que se estén utilizando y su potencial capacidad para modificar la microflora oral. El evaluar las vías de administración mas adecuadas para efecto terapéutico y benéfico en la cavidad oral en pacientes de alto riesgo con alta carga bacteriana.
Como se señala en estudios realizados por Meurman, Teughels et al. y Haukioja et al. (2008), es necesario obtener datos para mostrar el efecto inhibidor de los probióticos sobre la caries dental.

\section{CONCLUSIONES}

Se observaron diferencias significativas sobre el uso de cuatro concentraciones distintas de probióticos comercializados en Chile que contienen dos cepas de Lactobacillus que inhiben al Streptococcus mutans, esto supone la disminución de la colonización de dichas bacterias con el uso de estos probióticos, ya que modifican uno de los factores productores de la caries dental correspondiente a los microorganismos que actúan y son parte de su producción, se piensa que estas bacterias actúan inhibiendo el crecimiento ya que a través de la competencia por el sitio de colonización no permiten la llegada del Streptococcus mutans.

El desarrollo y demostración de que algunas cepas probióticas como Lactobacillus casei variedad rhamnosus (LCR35) y Lactobacillus johnsonii (LA1) disminuyen la colonización de las principales bacterias productoras de caries dental, pueden ser utilizados como apoyo en la prevención y profilaxis de la enfermedad en pacientes de alto riesgo cariogénico, como ayuda en la prevención como: el uso de fluoruros, promoción de higiene oral o cambios en el consumo de carbohidratos, proporcionando efectos favorables para la salud, especialmente en pacientes pediátricos, ya que son de fácil consumo y de un bajo costo.

REBOLLEDO, M.; ROJAS, E. \& SALGADO, F. Effect of two probiotics containing Lactobacillus rhamnosus and Lactobacillus johnsonii variety on the in vitro growth of Streptococcus mutans. Int. J. Odontostomat., 7(3):415-419, 2013.

ABSTRACT: Dental caries is one of the most prevalent diseases in the world and even more in the population of developing countries, so it is necessary to use new measures for prevention. Previous studies indicate that the effect of probiotic strains inhibit colonization of pathogens responsible for dental caries such as Streptococcus mutans, preventing the onset of dental caries. The aim was to measure the effect of the probiotic strains Lactobacillus casei variety rhamnosus (LCR32) and Lactobacillus johnsonii (LA1) on in vitro growth will Streptococcus mutans. We measured the effect in vitro of two probiotic strains marketed in Chile; Lactobacillus casei variety rhamnosus (LCR32) contained in Lactyl $®$ and Lactobacillus johnsonii (LA1) contained in Chamyto on in vitro growth of Streptococcus mutans. Culture media were made selective for Streptococcus mutans to which were added four different dilutions of each probiotic and measured the zone of inhibition of Streptococcus mutans with a foot tube. The probiotic strains Lactobacillus casei variety rhamnosus (LCR32) and Lactobacillus johnsonii (LA1) inhibit the growth of Streptococcus mutans. Both probiotics in the two higher concentrations no significant differences in relation to the zones of inhibition. However, the probiotic strain Lactobacillus casei rhamnosus variety (LCR35) showed the most significant zones of inhibition compared to the strain Lactobacillus johnsonii (LA1). The probiotic strains Lactobacillus casei rhamnosus variety (LCR35) and Lactobacillus Johnosonii (LA1) decreased the colonization of the main dental caries producing bacteria, so these probiotic could be used as support in the prevention and prophylaxis of the disease in high-risk cariogenic patients, additionally in other means of prevention.

KEYWORDS: caries, probiotic, bacterial strains, Lactobacillus, Streptococcus mutans. 
REBOLLEDO, M.; ROJAS, E. \& SALGADO, F. Efecto de dos probióticos que contienen cepas de Lactobacillus casei variedad rhamnosus y Lactobacillus johnsonii sobre el crecimiento in vitro de Streptococcus mutans. Int. J. Odontostomat., 7(3):415-419, 2013.

\section{REFERENCIAS BIBLIOGRÁFICAS}

Ahola, A. J.; Yli-Knuuttila, H.; Suomalainen, T.; Poussa, T.; Ahlström, A.; Meurman, J. H. \& Korpela, R. Short-term consumption of probiotic-containing cheese and its effect on dental caries risk factors. Arch. Oral Biol., 47(11):799-804, 2002.

Araya, M.; Morelli, L.; Reid, G.; Sanders, M. E.; Stanton, C.; Pineiro, M. \& Ben Embarek, P. Guidelines for the evaluation of probiotics in food. Report of a Joint FAO/ WHO Working Group on drafting guidelines for the evaluation of probiotics in food, London, Ontario, Canada. Rome, Geneva, Food and Agriculture Organization of the United Nations and World Health Organization, 2002.

Caglar, E.; Cildir, S. K.; Ergeneli, S.; Sandalli, N. \& Twetman, S. Salivary mutans streptococci and lactobacilli levels after ingestion of the probiotic bacterium Lactobacillus reuteri ATCC 55730 by straws or tablets. Acta Odontol. Scand., 64(5):314-8, 2006.

Caglar, E.; Kargul, B. \& Tanboga, I. Bacteriotherapy and probiotics' role on oral health. Oral Dis., 11(3):131-7, 2005.

Cona, T. E. Condiciones para un buen estudio de susceptibilidad mediante test de difusión en agar. Rev. Chil. Infectol., 19(2):77-81, 2002.

Haukioja, A.; Loimaranta, V. \& Tenovuo, J. Probiotic bacteria affect the composition of salivary pellicle and streptococcal adhesion in vitro. Oral Microbiol. Immunol., 23(4):336-43, 2008.

Meurman, J. H. Probiotics: do they have a role in oral medicine and dentistry? Eur. J. Oral Sci., 113(3):188-96, 2005.

Meurman, J. H. \& Stamatova, I. Probiotics: contributions to oral health. Oral Dis., 13(5):443-51, 2007.

Nikawa, H.; Makihira, S.; Fukushima, H.; Nishimura, H.; Ozaki, Y.; Ishida, K.; Darmawan, S.; Hamada, T.; Hara, K.; Matsumoto, A.; Takemoto, T. \& Aimi, R. Lactobacillus reuteri in bovine milk fermented decreases the oral carriage of mutans streptococci. Int. J. Food Microbiol., 95(2):219-23, 2004.

Pérez-Luyo, A. Probióticos: ¿una nueva alternativa en la prevención de la caries dental? Rev. Estomatol. Herediana, 18(1):65-8, 2008.

Teughels, W.; Van Essche, M.; Sliepen, I. \& Quirynen, M. Probiotics and oral healthcare. Periodontol. 2000, 48:111-47, 2008.
Twetman, S. \& Stecksén-Blicks, C. Probiotics and oral health effects in children. Int. J. Paediatr. Dent., 18(1):3-10, 2008.

Dirección para Correspondecia:

Mariana Rebolledo C.

Facultad de Odontología

Universidad del Desarrollo

Concepción

CHILE

Email: mariana.rebolledoc@gmail.com

Recibido : 18-10-2012

Aceptado: 09-10-2013 